\title{
BAYESIAN FUSION OF ASYNCHRONOUS INERTIAL, SPEED AND POSITION DATA FOR OBJECT TRACKING
}

\author{
Jiaming Liang and Simon Godsill
}

\author{
Signal Processing and Communication Laboratory, University of Cambridge, Cambridge, UK
}

\begin{abstract}
In this paper we present Bayesian methods for tracking scenarios in which an intrinsic coordinate model is considered and inertial measurements plus occasional position fixes are available. The methods are first tested using synthetic data, giving a comprehensive evaluation as to their performance. Further evaluation on real data also reveals our approaches can be favourable alternatives to existing inertial tracking/navigation models.
\end{abstract}

Index Terms - Bayesian tracking, sequential Monte Carlo, inertial measurements, sensor fusion

\section{INTRODUCTION}

Research in target tracking has been active over many decades. The ultimate goal of this topic is to estimate kinematic states as accurately as possible given noisy and limited measurements obtained from different sources. As surveyed in [1], even though there have been many successful tracking approaches that are based on whitenoise driven models, there are many mathematical models which are still underdeveloped. Among them, the intrinsic coordinate model (sometimes referred to as a curvilinear model, see [2]) has been shown to work very well in the cases of tracking manoeuvring objects $[3,4,5]$. Since the dynamics of an object can be expressed according to its intrinsic body frame, this model offers a promising solution to address the problem of fusing measurements from inertial sensors, which are necessarily in the object's body frame.

Closely coupled with the choice of a good model for target dynamics, inference algorithms are of particular importance when developing successful tracking applications. Among these, Sequential Monte Carlo (SMC) approaches [6, 7] using Importance Sampling (IS) have proved to be a powerful methodology when dealing with non-linear and non-Gaussian problems. On the other hand, Sequential Markov Chain Monte Carlo (SMCMC), as seen in [8, 9, 10, 11], is becoming known as a strong competitor for the IS-based methods in challenging scenarios. In contrast to IS-based SMC methods that can perform poorly in high dimensions, SMCMC has shown stronger potential $[12,13]$. Also, the properties of SMCMC methods can be desirable when developing algorithms for sequential batch inference, as shown in this paper.

Here we present intrinsic model based sequential batch inference methods, aiming at providing effective alternative solutions for tracking problems with inertial sensors. The methods are direct developments of the algorithm introduced in [5]. Specifically, we present novel state space models capable of incorporating acceleration (for the first time), speed, gyroscope and intermittent Cartesian position measurements and show how to deal with multiple flows of asynchronous measurements. A relevant work can be found in

Thanks to Cambridge Trust and China Scholarship Council for funding.
[14] where a multi-rate particle filter is proposed to deal with unsynchronised sensor measurements, though here we treat the state process in continuous time to solve the asynchronicity, as in [5]. Efficient section-wise forward-filtering-backward-sampling (FFBS) based kernels are designed and implemented by IS-based SMC filters and SMCMC (for the first time). In addition, to improve the efficacy of the generic SMCMC a parallel SMCMC algorithm is proposed. Finally, results with real vehicle data show the strong potential of the SMCMC methods.

\section{DYNAMIC AND MEASUREMENT MODELS}

The intrinsic coordinate based dynamic model, as in [4, 5], is a continuous time model, defined by

$$
\begin{aligned}
& a_{P}=\frac{T_{P}}{m}=s \dot{\psi}, \\
& a_{T}=\dot{s}=-\frac{\lambda}{m} s+\frac{T_{T}}{m},
\end{aligned}
$$

with the tangential acceleration $a_{T}$, the perpendicular acceleration $a_{P}$, the weight of a point mass $m$, the speed $s$, the heading rate $\dot{\psi}$ and a damping factor $\lambda$. By assuming that the forces $\left(T_{T}\right.$ and $\left.T_{P}\right)$ are piecewise constant between consecutive timestamps, the following equation can be obtained:

$$
s_{t}=e^{\frac{-\Delta \tau \lambda}{m}} s_{t-1}+\frac{T_{T}}{\lambda}\left(1-e^{\frac{-\Delta \tau \lambda}{m}}\right),
$$

where $\Delta \tau$ is time between $t$ and $t-1$ (this may in practice be variable for some sensor types). From Eqns. (1) - (3) it is clear that once $s_{t}, s_{t-1}$ and $\dot{\psi}_{t}$ are given, $T_{T}\left(s_{t}, s_{t-1}\right)$ is deterministic, so is $T_{P}\left(\dot{\psi}_{t}, s_{t}\right)$. We also propose to use a state variable $\boldsymbol{\alpha}_{t}=\left[\dot{\psi}_{t}, b_{t}\right]^{T}$ in which $b_{t}$ is a gyroscope bias term that is modelled as a Wiener random process. Provided that we have normal densities for $T_{T} \sim$ $\mathcal{N}\left(\mu_{T}, \sigma_{T}^{2}\right)$ and $T_{P} \sim \mathcal{N}\left(0, \sigma_{P}^{2}\right)$, the state transition densities for $s$ and $\boldsymbol{\alpha}$ are given by

$$
\begin{gathered}
p\left(s_{t+1} \mid s_{t}\right)=\mathcal{N}\left(s_{t+1} \mid e^{-\frac{\Delta \tau \lambda}{m}} s_{t}+\frac{\mu_{T}}{\lambda}\left(1-e^{-\frac{\Delta \tau \lambda}{m}}\right),\right. \\
\left.\frac{\sigma_{T}^{2}}{\lambda^{2}}\left(1-e^{-\frac{\Delta \tau \lambda}{m}}\right)^{2}\right) \\
p\left(\boldsymbol{\alpha}_{t+1} \mid \boldsymbol{\alpha}_{t}, s_{t+1}\right)=\mathcal{N}\left(\boldsymbol{\alpha}_{t+1} \mid A \boldsymbol{\alpha}_{t}, C\right) .
\end{gathered}
$$

with $A=\left[\begin{array}{ll}0 & 0 \\ 0 & 1\end{array}\right]$ and $C=\operatorname{diag}\left(\left[\frac{\sigma_{P}^{2}}{m^{2} s_{t+1}^{2}}, \sigma_{b}^{2} \Delta \tau\right]\right)$. Furthermore, in terms of Eqns. (1) - (3) the accelerations can be obtained as $a_{P, t}=$ $s_{t} \dot{\psi}_{t}$ and $a_{T, t}=c\left(s_{t}-s_{t-1}\right)$ where $c=\lambda\left(e^{-\frac{\Delta \tau \lambda}{m}}\right) / m(1-$ $\left.e^{-\frac{\Delta \tau \lambda}{m}}\right)$. One of the reasons of choosing an intrinsic frame dynamic model is that the body frames of inertial sensors (e.g. accelerometers, gyroscopes and speedometers) are aligned with the object's 
intrinsic coordinate (body) frame. This results in linear models for the kinematic states in the body frame connecting the dynamics to the inertial measurements, which later allows us to propose states effectively using Kalman filtering-based methods.

Given that the inertial sensors are fixed in the intrinsic frame of an object, which is a practical scenario for ground vehicles, we can now extend the models in [5] and construct the following measurement models for accelerometer, speedometer and gyroscope measurements:

$$
\begin{aligned}
Y_{\boldsymbol{\alpha}, t} & =\left[\begin{array}{c}
\hat{\dot{\psi}}_{t} \\
\hat{a}_{P, t}
\end{array}\right]=\left[\begin{array}{cc}
1 & 1 \\
s_{t} & 0
\end{array}\right] \boldsymbol{\alpha}_{t}+\boldsymbol{v}_{\boldsymbol{\alpha}, t}, \\
\boldsymbol{v}_{\boldsymbol{\alpha}, t} & \sim \mathcal{N}\left(0, \Sigma_{\boldsymbol{\alpha}}\right), \quad \Sigma_{\boldsymbol{\alpha}}=\operatorname{diag}\left(\left[\sigma_{\hat{\hat{\psi}}}^{2}, \sigma_{\hat{a}_{P}}^{2}\right]\right), \\
Y_{s, t} & =\left[\begin{array}{c}
\hat{a}_{T, t} \\
\hat{s}_{t}
\end{array}\right]=\left[\begin{array}{cc}
c & -c \\
1 & 0
\end{array}\right]\left[\begin{array}{c}
s_{t} \\
s_{t-1}
\end{array}\right]+\boldsymbol{v}_{s, t}, \\
\boldsymbol{v}_{s, t} & \sim \mathcal{N}\left(0, \Sigma_{s}\right), \quad \Sigma_{s}=\operatorname{diag}\left(\left[\sigma_{\hat{a}_{T}}^{2}, \sigma_{\hat{s}}^{2}\right]\right) .
\end{aligned}
$$

Here $\hat{\dot{\psi}}$ is the measurement of angular velocity around the $z$-axis of a gyroscope while $\hat{s}$ is the speedometer measurement in the forward direction. As for accelerations, we use $\hat{a}_{T}$ and $\hat{a}_{P}$ to represent measurements on the $x$-axis (forward) and the $y$-axis (leftward) of an accelerometer. In addition to inertial measurements, the model for intermittent position information is given by

$$
f\left(\hat{\boldsymbol{z}}_{t} \mid \boldsymbol{z}_{t}\right)=\mathcal{N}\left(\hat{\boldsymbol{z}}_{t} \mid \boldsymbol{z}_{t}, \Sigma_{\boldsymbol{z}}\right), \quad \Sigma_{\boldsymbol{z}}=\operatorname{diag}\left(\left[\sigma_{x}^{2}, \sigma_{y}^{2}\right]\right),
$$

where the position $\boldsymbol{z}_{t}=\left[x_{t}, y_{t}\right]^{T}$ in Cartesian coordinates is calculated based on speeds and heading rates up to time $t$, as in [5].

\section{INFERENCE ALGORITHMS}

\subsection{Sequential Importance Resampling (SIR)}

Since the introduction in [15], SIR, also known as particle filtering, has become a useful approach to solving intractable inference problems, in contrast to linear Kalman methods. To use SIR in this setting first factorise the posterior distribution as follows, with batch size $k \geq 1$ defined as the section length between two consecutive position measurements,

$$
\begin{aligned}
& \pi\left(s_{0: t}, \boldsymbol{\alpha}_{0: t} \mid Y_{\boldsymbol{\alpha}, 0: t}, Y_{s, 0: t}, \hat{\boldsymbol{z}}_{0: t}\right) \\
\propto & p\left(s_{0: t}, \boldsymbol{\alpha}_{0: t} \mid Y_{\boldsymbol{\alpha}, 0: t}, Y_{s, 0: t}, \hat{\boldsymbol{z}}_{0: t-k}\right) p\left(\hat{\boldsymbol{z}}_{t} \mid \boldsymbol{\alpha}_{0: t}, s_{0: t}\right) \\
\propto & p\left(s_{0: t-k}, \boldsymbol{\alpha}_{0: t-k} \mid Y_{\boldsymbol{\alpha}, 0: t-k}, Y_{s, 0: t-k}, \hat{\boldsymbol{z}}_{0: t-k}\right) \\
& \times p\left(s_{t-k+1: t} \mid Y_{s, t-k+1: t}, s_{t-k}\right) p\left(Y_{s, t-k+1: t} \mid s_{t-k}\right) \\
& \times p\left(\boldsymbol{\alpha}_{t-k+1: t} \mid Y_{\boldsymbol{\alpha}, t-k+1: t}, s_{t-k+1: t}, \boldsymbol{\alpha}_{t-k}\right) \\
& \times p\left(Y_{\boldsymbol{\alpha}, t-k+1: t} \mid s_{t-k+1: t}, \boldsymbol{\alpha}_{t-k}\right) p\left(\hat{\boldsymbol{z}}_{t} \mid \boldsymbol{\alpha}_{0: t}, s_{0: t}\right)
\end{aligned}
$$

The dependency on states at time $t-k$ is a direct result from the Markovian assumption. Suppose now that drawing samples from importance distributions $p\left(s_{t-k+1: t} \mid Y_{s, t-k+1: t}, s_{t-k}\right)$ and $p\left(\boldsymbol{\alpha}_{t-k+1: t} \mid Y_{\boldsymbol{\alpha}, t-k+1: t}, s_{t-k+1: t}, \boldsymbol{\alpha}_{t-k}\right)$ is feasible, the weight updating equation for SIR is given by

$$
\begin{aligned}
\tilde{\omega}_{t}^{(i)}= & \frac{\pi\left(s_{0: t}, \boldsymbol{\alpha}_{0: t} \mid Y_{\boldsymbol{\alpha}, 0: t}, Y_{s, 0: t}, \hat{\boldsymbol{z}}_{0: t}\right)}{q\left(s_{0: t}, \boldsymbol{\alpha}_{0: t} \mid Y_{\boldsymbol{\alpha}, 0: t}, Y_{s, 0: t}\right)} \\
= & \tilde{\omega}_{t-k}^{(i)} p\left(Y_{s, t-k+1: t} \mid s_{t-k}^{(i)}\right) p\left(\hat{\boldsymbol{z}}_{t} \mid \boldsymbol{\alpha}_{0: t}^{(i)}, s_{0: t}^{(i)}\right) \\
& \times p\left(Y_{\boldsymbol{\alpha}, t-k+1: t} \mid s_{t-k+1: t}^{(i)}, \boldsymbol{\alpha}_{t-k}^{(i)}\right) .
\end{aligned}
$$

The remaining question is about how to sample from the proposals as well as to compute the likelihood terms in the weight updating equation. To do this, we adopt the idea of forward-filteringbackward-sampling (FFBS) [16, 17, 18]. Specifically, for speed the forward filtering (FF) step updates $p\left(s_{t-1: t} \mid Y_{s, t-k+1: t}, s_{t-k}\right)$ to $p\left(s_{t: t+1} \mid Y_{s, t-k+1: t+1}, s_{t-k}\right)$ in the light of a new measurement $Y_{s, t+1}$ recursively. More precisely, in the FF prediction stage the speeds are updated according to the dynamical model:

$$
\begin{aligned}
& p\left(s_{t: t+1} \mid Y_{s, t-k+1: t}, s_{t-k}\right) \\
= & \int p\left(s_{t-1: t} \mid Y_{s, t-k+1: t}, s_{t-k}\right) p\left(s_{t+1} \mid s_{t}\right) d s_{t-1} .
\end{aligned}
$$

Then the predictive distribution can be corrected when new measurements become available:

$$
\begin{aligned}
& p\left(s_{t: t+1} \mid Y_{s, t-k+1: t+1}, s_{t-k}\right) \\
\propto & p\left(s_{t: t+1} \mid Y_{s, t-k+1: t}, s_{t-k}\right) p\left(Y_{s, t+1} \mid s_{t: t+1}\right) .
\end{aligned}
$$

Backward sampling (BS) is applied to drawing samples from the joint state distribution conditioned on all observations:

$$
\begin{aligned}
p\left(s_{t-k+1: t} \mid Y_{s, t-k+1: t}, s_{t-k}\right) & =p\left(s_{t}, s_{t-1} \mid Y_{s, t-k+1: t}, s_{t-k}\right) \\
& \times \prod_{l=t-2}^{t-k+1} p\left(s_{l} \mid s_{l+1}, Y_{s, t-k+1: l+1}, s_{t-k}\right)
\end{aligned}
$$

where

$$
\begin{array}{r} 
\\
p\left(s_{t} \mid s_{t+1}, Y_{s, t-k+1: t+1}, s_{t-k}\right) \\
\propto p\left(s_{t}, s_{t+1} \mid Y_{s, t-k+1: t+1}, s_{t-k}\right) .
\end{array}
$$

Last, the likelihood term $p\left(Y_{s, t-k+1: t} \mid s_{t-k}\right)$ can be calculated via Prediction Error Decomposition (PED):

$$
\begin{aligned}
p\left(Y_{s, t-k+1: t} \mid s_{t-k}\right)=p\left(Y_{s, t-k+1} \mid s_{t-k}\right) & \\
\times & \prod_{l=t-k+2}^{t} p\left(Y_{s, l} \mid Y_{s, t-k+1: l-1}, s_{t-k}\right),
\end{aligned}
$$

with

$$
\begin{gathered}
p\left(Y_{s, t} \mid Y_{s, t-k+1: t-1}, s_{t-k}\right)=\int p\left(Y_{s, t} \mid s_{t-1}, s_{t}\right) \\
\quad \times p\left(s_{t-1}, s_{t} \mid Y_{s, t-k+1: t-1}, s_{t-k}\right) d s_{t-1} d s_{t} .
\end{gathered}
$$

Similar Kalman style scheme can be adopted to draw samples from $p\left(\boldsymbol{\alpha}_{t-k+1: t} \mid Y_{\boldsymbol{\alpha}, t-k+1: t}, s_{t-k+1: t}, \boldsymbol{\alpha}_{t-k}\right)$. Note although the problem is formalised in a sequential batch (or section-wise) fashion, the introduced methods also apply to strictly sequential case where $k=1$. In such a case the proposals degenerate to locally optimal kernels conditioned on the new measurements and the previous state.

\subsubsection{Resample-move (RM)}

Resample-move has been a common improvement technique for the classical particle filters [19]. The mechanism behind RM is rejuvenating degenerated particles after the resampling stage so as to improve the empirical approximation obtained by SIR. Conditioned on the states up to time $t-k$ and all measurements, the target distribution in the RM step is expressed as

$$
p\left(s_{t-k+1: t}, \boldsymbol{\alpha}_{t-k+1: t} \mid s_{0: t-k}, \boldsymbol{\alpha}_{0: t-k}, Y_{s, 0: t}, Y_{\boldsymbol{\alpha}, 0: t}, \hat{\boldsymbol{z}}_{0: t}\right) .
$$


A Metropolis-Hastings (MH) [20] kernel can be designed to propose $\left\{s_{t-k+1: t}^{*}, \boldsymbol{\alpha}_{t-k+1: t}^{*}\right\}$ with proposals the same as those used in SIR. The resulting acceptance ratio is

$$
\begin{aligned}
\rho_{R M}= & \min \left(1, f\left(\frac{p\left(Y_{\boldsymbol{\alpha}, t-k+1: t} \mid s_{t-k+1: t}^{*}, \boldsymbol{\alpha}_{t-k}^{(i)}\right)}{p\left(Y_{\boldsymbol{\alpha}, t-k+1: t} \mid s_{t-k+1: t}^{(i)}, \boldsymbol{\alpha}_{t-k}^{(i)}\right)}\right.\right. \\
& \left.\left.\frac{p\left(\hat{\boldsymbol{z}}_{t} \mid s_{0: t-k}^{(i)}, \boldsymbol{\alpha}_{0: t-k}^{(i)}, s_{t-k+1: t}^{*}, \boldsymbol{\alpha}_{t-k+1: t}^{*}\right)}{p\left(\hat{\boldsymbol{z}}_{t} \mid s_{0: t-k}^{(i)}, \alpha_{0: t-k}^{(i)}, s_{t-k+1: t}^{(i)}, \boldsymbol{\alpha}_{t-k+1: t}^{(i)}\right)}\right)\right) .
\end{aligned}
$$

Since each selected particle, $\left\{s_{0: t}^{(i)}, \boldsymbol{\alpha}_{0: t}^{(i)}\right\}$, before being moved, is already approximately distributed according to $\pi_{0: t}$, the usual burn-in period for MCMC is not necessary, as suggested in [19].

\subsection{Sequential Markov Chain Monte Carlo (SMCMC)}

The adopted SMCMC algorithm, as in most existing SMCMC approaches $[10,11,12,13]$, follows a mixture sampling based procedure that selects a joint draw action with probability $P_{J}$ which updates all states simultaneously and refinement steps with probability $1-P_{J}$ in which $\left\{s_{0: t-k}, \boldsymbol{\alpha}_{0: t-k}\right\}$ and $\left\{s_{t-k+1: t}, \boldsymbol{\alpha}_{t-k+1: t}\right\}$ are updated individually.

\subsubsection{Jointly drawing $\left\{\boldsymbol{\alpha}_{0: t}, s_{0: t}\right\}$}

To make a joint draw with a target distribution defined in Eqn. (9) at the $m^{\text {th }}$ MCMC iteration, two steps are required: first draw $\left\{\boldsymbol{\alpha}_{0: t-k}^{*}, s_{0: t-k}^{*}\right\}$ using the empirical particle representation obtained at time $t-k$ :

$$
\begin{aligned}
& q\left(\boldsymbol{\alpha}_{0: t-k}, s_{0: t-k} \mid \boldsymbol{\alpha}_{0: t-k}^{m-1}, s_{0: t-k}^{m-1}\right) \\
= & \hat{p}\left(s_{0: t-k}, \boldsymbol{\alpha}_{0: t-k} \mid Y_{\boldsymbol{\alpha}, 0: t-k}, Y_{s, 0: t-k}, \hat{\boldsymbol{z}}_{0: t-k}\right) .
\end{aligned}
$$

This is then followed by the proposal of $\left\{\boldsymbol{\alpha}_{t-k+1: t}^{*}, s_{t-k+1: t}^{*}\right\}$ conditioned on the states drawn in the first step:

$$
\begin{aligned}
q & \left(\boldsymbol{\alpha}_{t-k+1: t}, s_{t-k+1: t} \mid \boldsymbol{\alpha}_{t-k+1: t}^{m-1}, s_{t-k+1: t}^{m-1}\right) \\
=p & \left(\boldsymbol{\alpha}_{t-k+1: t} \mid Y_{\boldsymbol{\alpha}, t-k+1: t}, s_{t-k+1: t}, \boldsymbol{\alpha}_{t-k}^{*}\right) \\
& \times p\left(s_{t-k+1: t} \mid Y_{s, t-k+1: t}, s_{t-k}^{*}\right) .
\end{aligned}
$$

As a result, the acceptance ratio for a $\mathrm{MH}$ kernel is obtained as follows,

$$
\begin{aligned}
& \rho_{1}=\min \left(1, f\left(\frac{p\left(Y_{\boldsymbol{\alpha}, t-k+1: t} \mid s_{t-k+1: t}^{*}, \boldsymbol{\alpha}_{t-k}^{*}\right)}{p\left(Y_{\boldsymbol{\alpha}, t-k+1: t} \mid s_{t-k+1: t}^{m-1}, \boldsymbol{\alpha}_{t-k}^{m-1}\right)}\right.\right. \\
& \left.\left.\frac{p\left(Y_{s, t-k+1: t} \mid s_{t-k}^{*}\right) p\left(\hat{\boldsymbol{z}}_{t} \mid \boldsymbol{\alpha}_{0: t}^{*}, s_{0: t}^{*}\right)}{p\left(Y_{s, t-k+1: t} \mid s_{t-k}^{m-1}\right) p\left(\hat{\boldsymbol{z}}_{t} \mid \boldsymbol{\alpha}_{0: t}^{m-1}, s_{0: t}^{m-1}\right)}\right)\right) .
\end{aligned}
$$

\subsubsection{Refining $\left\{s_{0: t-k}, \boldsymbol{\alpha}_{0: t-k}\right\}$ and $\left\{s_{t-k+1: t}, \boldsymbol{\alpha}_{t-k+1: t}\right\}$}

Coupled with the joint proposal, a series of Metropolis-within-Gibbs steps can be carried out to refine states respectively. More specifically, we first refine the estimate of $\left\{s_{0: t-k}, \boldsymbol{\alpha}_{0: t-k}\right\}$ using a MH kernel with the following target distribution:

$$
p\left(s_{0: t-k}, \boldsymbol{\alpha}_{0: t-k} \mid s_{t-k+1: t}, \boldsymbol{\alpha}_{t-k+1: t}, Y_{s, 0: t}, Y_{\alpha, 0: t}, \hat{z}_{0: t}\right)
$$
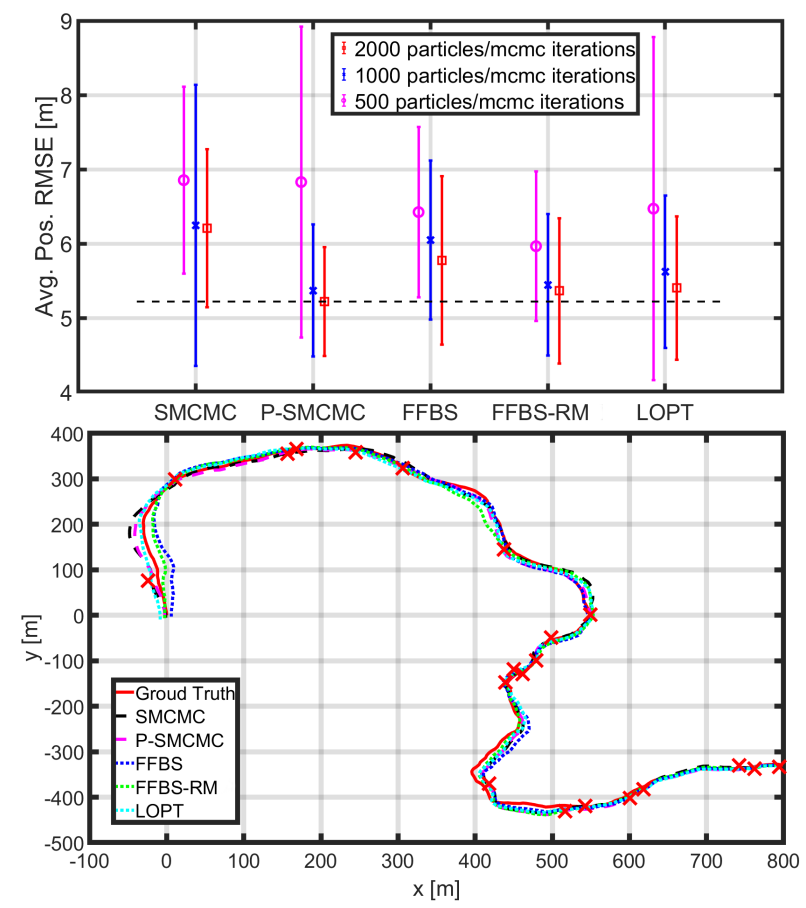

Fig. 1: Results on synthetic data. Top: Tracking performance of different algorithms on 100 datasets synthesised according to the intrinsic coordinate model. Bottom: An example of simulated trajectories and tracking results, with less position measurements (red cross, $\lambda_{P O I}=1 / 15$ ).

and a proposal identical to that given by Eqn. (17). $\left\{s_{0: t-k}^{*}, \boldsymbol{\alpha}_{0: t-k}^{*}\right\}$ is then accepted according to the following ratio:

$$
\begin{aligned}
\rho_{2}= & \min \left(1, f\left(\frac{p\left(\boldsymbol{\alpha}_{t-k+1}^{m-1} \mid \boldsymbol{\alpha}_{t-k}^{*}, s_{t-k+1}^{m-1}\right) p\left(s_{t-k+1}^{m-1} \mid s_{t-k}^{*}\right)}{p\left(\boldsymbol{\alpha}_{t-k+1}^{m-1} \mid \boldsymbol{\alpha}_{t-k}^{m-1}, s_{t-k+1}^{m-1}\right) p\left(s_{t-k+1}^{m-1} \mid s_{t-k}^{m-1}\right)}\right.\right. \\
& \left.\left.\frac{p\left(\hat{\boldsymbol{z}}_{t} \mid s_{0: t-k}^{*}, \boldsymbol{\alpha}_{0: t-k}^{*}, s_{t-k+1: t}^{m-1}, \boldsymbol{\alpha}_{t-k+1: t}^{m-1}\right)}{p\left(\hat{\boldsymbol{z}}_{t} \mid s_{0: t-k}^{m-1}, \boldsymbol{\alpha}_{0: t-k}^{m-1}, s_{t-k+1: t}^{m-1}, \boldsymbol{\alpha}_{t-k+1: t}^{m-1}\right)}\right)\right)
\end{aligned}
$$

The next step is refining $\left\{s_{t-k+1: t}, \boldsymbol{\alpha}_{t-k+1: t}\right\}$. To do this, see the RM target distribution as well as its associated acceptance ratio in Section 3.1.1. Note that after any acceptance event all likelihood terms need to be updated accordingly.

\subsubsection{Parallel SMCMC}

Generic SMCMC algorithms can still be inefficient when dealing with highly correlated variables in high dimensional systems. To account for this, we propose an intuitive but effective solution which runs multiple Markov chains in parallel. The idea is to provide greater diversity and to avoid the situation in which we only have one chain and it gets stuck at one ancestor trajectory. This algorithm also introduces a new parameter, which is the number of parallel chains $N_{\text {chain }}$.

\section{EVALUATION AND RESULTS}

\subsection{Synthetic data}

The proposed inference methods are firstly tested on 100 Monte Carlo realisations (i.e. 100 trajectories, each for 300 seconds) of 


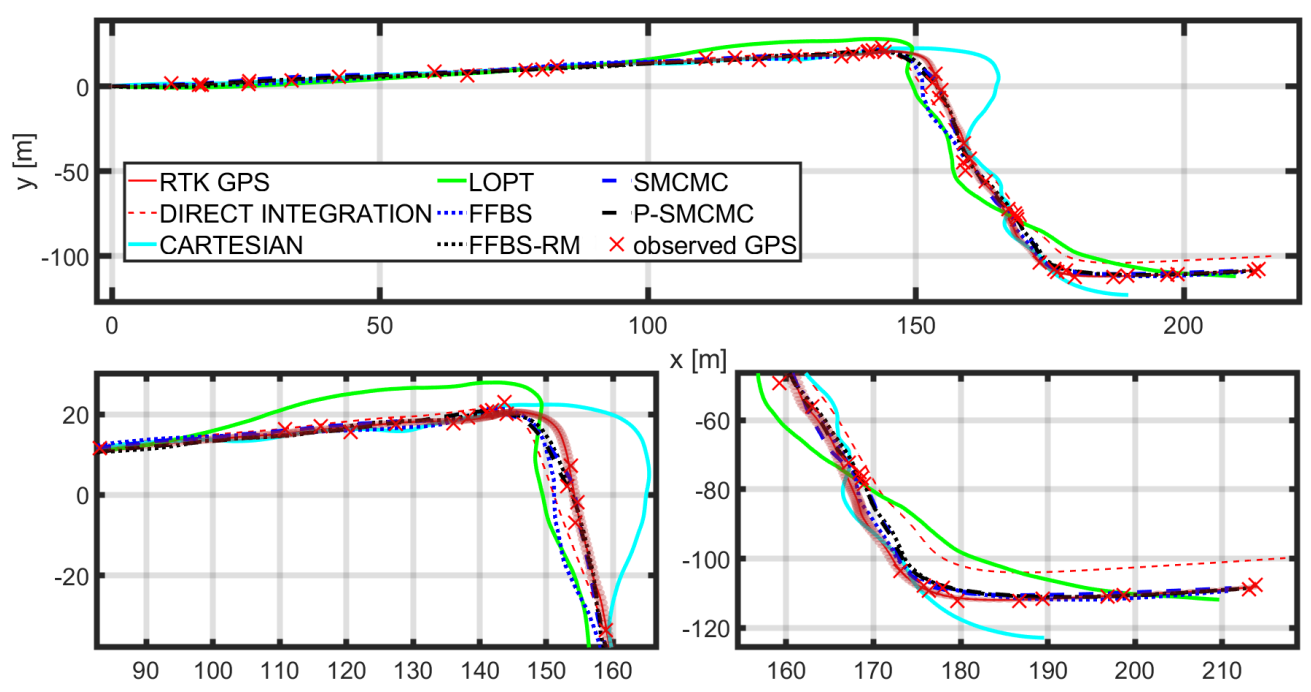

Fig. 2: Tracking performance across all algorithms on a KITTI dataset ["2011_09_26_drive_0117_sync”].

the problem with varied number of particles. In addition, a particle filter using locally optimal kernels (LOPT), as in [5], is implemented for comparison. In detail, the parameters used in the simulation are: $\mu_{T}=15, \sigma_{T}=30, \sigma_{P}=220, f_{s}=1 \mathrm{~Hz}, m=200$, $\lambda=3, \sigma_{b}=0.5^{\circ}, \sigma_{\hat{s}}=1, \sigma_{\hat{\psi}}=18^{\circ}, \sigma_{\hat{a}_{T}}=\sigma_{\hat{a}_{P}}=0.5$, $\sigma_{x}=\sigma_{y}=5, P_{J}=0.5$ and $N_{\text {chain }}=4$. The time of arrival of GPS signals is governed by a homogeneous Poisson process with intensity $\lambda_{\mathcal{P O I}}=1 / 6$. Moreover, the total number of iterations (including burn-in period) used in MCMC based algorithms is set to be the same as the particle number used in FFBS, FFBS-RM and LOPT. Note FFBS-RM actually requires twice as much as computational power $\left(N_{R M}=1\right)$ compared to the others as it has to do RM for each resampled particle.

The results of the average position RMSEs and their associated standard deviations are shown in Fig. 1, from which it is clear that the proposed parallel SMCMC (P-SMCMC) algorithm improves the tracking performance compared to the generic SMCMC. It also gives the best estimates with enough iterations. However, the performance of SMCMC based algorithms drops significantly when not running the chains for long enough time. As each parallel chain is run for just a quarter of the total number of iterations, P-SMCMC gives poor results with 500 iterations.

\subsection{Real Data}

KITTI [21] ground vehicle datasets are chosen to test our algorithms as they contain high precision RTK-GPS measurements which can be treated as ground truth. Fig. 2 shows tracking performance of different methods on a 66 -second dataset, given random initial heading angle (uniformly in $[0,2 \pi]$ ) and $\lambda_{P O I}=2 / 3$. The red shaded area along the RTK trajectory represents the precision of the ground truth position measurements. Additionally, a hybrid Cartesian model, which combines a constant-acceleration model (for $[x, \dot{x}, \ddot{x}, y, \dot{y}, \ddot{y}]$ ) with a constant-velocity model (for $[\psi, \dot{\psi}]$ ), and a direct integration (with correct initial heading) of the inertial measurements, are provided as baselines. The relatively poor performance of LOPT, especially when it is around the area without position measurements, highlights the fact that it is a 'one-shot' sequential algorithm. It cannot 'look ahead' to use coming position measurements as a guidance, which is a main difference between it

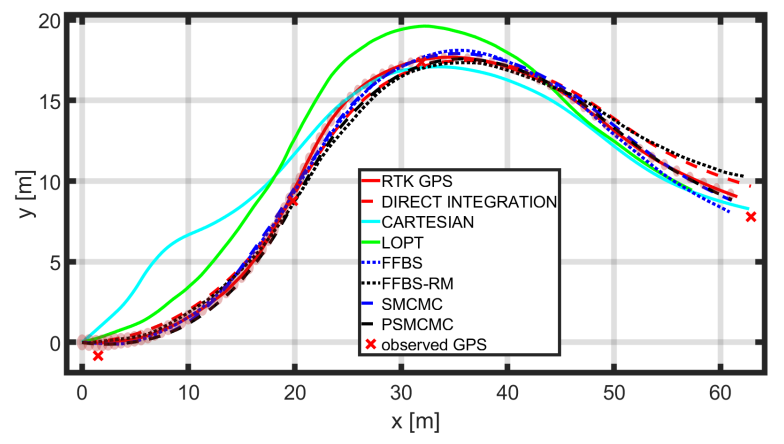

Fig. 3: Another KITTI dataset [“2011_09_26_drive_005_sync”], with merely 4 position observations.

and the other algorithms. Although enhancement can be achieved by running a particle smoother backwards many times, it is computationally expensive. On the contrary, FFBS-RM, SMCMC and P-SMCMC improve the proposed states and the retrospective performance while running in a sequential batch sense and maintaining relatively low computational cost. This characteristic becomes more prominent as in Fig. 3.

\section{CONCLUSIONS}

In this paper we show for the first time how to fuse inertial (accelerometer, gyroscope and speedometer) and asynchronous position measurements into an intrinsic coordinate model and to design corresponding Bayesian inference algorithms. Results on synthetic and real data have shown that a novel parallel chain based modification to the generic SMCMC helps improve performance. This also encourages further exploration on effects of using different numbers of parallel chains and introducing interaction between independent chains in P-SMCMC. 


\section{References}

[1] X. Li and V. Jilkov, "Survey of maneuvering target tracking. Part I. Dynamic models," IEEE Transactions on Aerospace and Electronic Systems, vol. 39, no. 4, pp. 1333-1364, Oct. 2003.

[2] R. Best and J. Norton, "A new model and efficient tracker for a target with curvilinear motion," IEEE Transactions on Aerospace and Electronic Systems, vol. 33, no. 3, pp. 10301037, July 1997.

[3] P. Bunch and S. Godsill, "Dynamical models for tracking with the variable rate particle filter," in Proc. International Conference on Information Fusion (FUSION'12), Singapore, 2012, pp. 1769-1775.

[4] S. Godsill, J. Vermaak, W. Ng and J. Li, "Models and algorithms for tracking of maneuvering objects using variable rate particle filters," Proceedings of the IEEE, vol. 95, no. 5, pp. 925-952, May 2007.

[5] J. Liang and S. Godsill, "A particle filter localisation system for indoor track cycling using an intrinsic coordinate model," in Proc. International Conference on Information Fusion (FUSION'18), July 2018, pp. 1896-1903.

[6] A. Doucet, S. Godsill and C. Andrieu, "On sequential Monte Carlo sampling methods for Bayesian filtering," Statistics and computing, vol. 10, no. 3, pp. 197-208, 2000.

[7] O. Cappé, S. Godsill and E. Moulines, "An overview of existing methods and recent advances in sequential Monte Carlo," Proceedings of the IEEE, vol. 95, no. 5, pp. 899-924, May 2007.

[8] C. Berzuini, N. Best, WR. Gilks and C. Larizza, "Dynamic conditional independence models and Markov Chain Monte Carlo methods," Journal of the American Statistical Association, vol. 92, no. 440, pp. 1403-1412, 1997.

[9] A. Golightly and D. J. Wilkinson, "Bayesian sequential inference for nonlinear multivariate diffusions," Statistics and Computing, vol. 16, no. 4, pp. 323-338, Dec 2006.

[10] F. Septier, S. Pang, S. Godsill and A. Carmi, "Tracking of coordinated groups using marginalised MCMC-based particle algorithm," in 2009 IEEE Aerospace conference, March 2009, pp. 1-11.

[11] S. Pang, J. Li and S. Godsill, "Detection and tracking of coordinated groups," IEEE Transactions on Aerospace and Electronic Systems, vol. 47, no. 1, pp. 472-502, Jan. 2011.

[12] L. Mihaylova, A. Carmi, F. Septier, A. Gning, S. Pang and S. Godsill, "Overview of Bayesian sequential Monte Carlo methods for group and extended object tracking," Digital Signal Processing, vol. 25, pp. 1 - 16, 2014.

[13] F. Septier and G. Peters, "Langevin and hamiltonian based sequential MCMC for efficient Bayesian filtering in highdimensional spaces," IEEE Journal of Selected Topics in Signal Processing, vol. 10, no. 2, pp. 312-327, Mar. 2016.

[14] R. Hostettler and P. M. Djurić, "Vehicle tracking based on fusion of magnetometer and accelerometer sensor measurements with particle filtering," IEEE Transactions on Vehicular Technology, vol. 64, no. 11, pp. 4917-4928, Nov 2015.
[15] N. Gordon, D. Salmond and A. Smith, "Novel approach to nonlinear/non-Gaussian Bayesian state estimation," in IEE Proceedings F (Radar and Signal Processing). IET, 1993, pp. 107-113.

[16] C. Carter and R. Kohn, "On Gibbs sampling for state space models," Biometrika, vol. 81, no. 3, pp. 541-553, Aug. 1994.

[17] S. Frühwirth-Schnatter, "Data augmentation and dynamic linear models," Journal of Time Series Analysis, vol. 15, no. 2, pp. 183-202, Mar. 1994.

[18] S. Hore, M. Johannes, H. Lopes, R. McColluch and N. Polson, "Bayesian computation in finance," in Frontiers of Statistical Decision Making and Bayesian Analysis, Ed. MH Chen, P. Müller, D. Sun and K. Ye, Eds., pp. 383-396. New York: Springer, Aug. 2010.

[19] WR. Gilks and C. Berzuini, "Following a moving target Monte Carlo inference for dynamic Bayesian models," Journal of the Royal Statistical Society: Series B (Statistical Methodology), vol. 63, no. 1, pp. 127-146, 2001.

[20] C. P. Robert and G. Casella, Monte Carlo statistical methods, New York: Springer, 2004.

[21] A. Geiger, P. Lenz, C. Stiller and R. Urtasun, "Vision meets robotics: The KITTI dataset," International Journal of Robotics Research, vol. 32, no. 11, pp. 1231-1237, Sept. 2013. 